\title{
Comparative Study on the Effect of Two Different Sequential Combination Training Methods on Fat Reduction
}

\author{
Li Li \\ Chongqing University, Chongqing 400044, China \\ DOI: $10.32629 /$ jher.v2i6.570
}

\begin{abstract}
In this paper, overweight or obese young women with sports experience and body fat rate $>30 \%$ were divided into groups to compare the effect of "HIIT" and "aerobic" exercise in different sequence combination training methods, and analyze whether "HIIT + Aerobic" is more effective than "aerobic + HIIT". The aim is to provide more effective training programs and establish scientific fitness awareness for overweight or obese young women who have sports experience.

Keywords: HIIT, aerobic exercise, integrated training, fat loss, FMS
\end{abstract}

\section{Introduction}

With the continuous improvement of people's living standard, obesity has become a chronic disease all over the world. The low level of cardiopulmonary function and high body fat percentage (\%) caused by obesity are the root cause of many chronic diseases. Therefore, how to improve the body composition of obese adults and prevent diseases is a topic that most people care about at present. In terms of exercise fat reduction methods, studies have shown that HIT not only improves and enhances the cardiovascular function of the body, but also has the effect of fat reduction. HIIT is a high-intensity exercise method. From the perspective of preventing joint function injury and maximizing fat loss benefits, it is a problem to study whether there is difference in training effect between obese people with and without exercise experience. At the same time, "aerobic" and "HIIT" should be combined in different order to see which combination form is better, which is also needed to be explored in this paper.

On the basis of previous studies, the author made further explorations with aspirations to lay a theoretical foundation for the establishment of scientific and systematic training methods for fat reduction and body shaping. A comparative study was conducted on the sequence of "HIIT" and "aerobic" exercise, to explore the effects of different combination training methods on overweight or mildly obese women, to seek the practical effects of the two and the best exercise methods, to provide a basis for scientific exercise for fat reduction people, and to develop a set of simple and easy to promote exercise prescription.

\section{Research design}

\subsection{Research objects}

The research objects of this paper are all young women in Chongqing University (within the age range of 22-40), and the requirements are as follows:

(1) Having training experience, irregular exercise for nearly 3 months, healthy and stable weight;

(2) FMS $\geq 14$ and body fat percentage $>30 \%$;

(3) No other sports contraindications.

\subsection{Research methods}

\subsubsection{Literature method}

By collecting and sorting out the literature on HIIT-related fat loss of aerobic exercise, we studied and analyzed it, and formed a scientific cognition of the research topic. This paper mainly collects literature materials through Plumbed database, CNKI, sports papers, sports journals, magazines and textbooks. According to the research task, the focus of reading related exercise physiology, exercise training, FMS screening, physical fitness and nutrition related data. These literature and materials have played an important role in promoting the completion of this research, for understanding the basic macro process of training, prevention of joint function and sports injury, development of plastic training methods of fat reduction.

\subsubsection{Experiment method}

(1) Experimental subjects

Ten young women with good joint function and no other diseases affecting exercise were determined by FMS, physical master body composition tester and other tests. 
The 10 qualified subjects were divided into 2 groups with 5 subjects in each group. Group A was the experimental group, which adopted HIIT followed by aerobics, i.e. "HIIT+ aerobics", and the author and A teaching assistant were responsible for it. Group B was the contrast group, which was first aerobic followed by HIIT, i.e., "aerobic +HIIT". The author's two fellow researchers were in charge.

(2) Test indicators and methods

1) Test indicators

A. Body shape detection: Height, weight, BMI, waistline, hipline, waist-hip ratio

B. Body composition: body fat percentage and lean body weight

C. Cardiopulmonary function: quiet heart rate

D. FMS screening tests: To assess flexibility and stability of the shoulder, spine, hip, knee, and ankle joints

2) Test method

A. Body shape test indicators

Test instrument: soft ruler, master body composition tester

The test method

Weight: Remind the tester not to wear relevant metal accessories (e.g. necklace) before the test. After entering the subject's number, the subject will remove his/her shoes and stand upright on the instrument. Complete the test with the instrument at eye level, remain silent during the test, do not shake or move the body, and record the weight test results

BMI: BMI $=$ weight/height $(\mathrm{m} 2)$

Waist: Keep your body upright and your arms down naturally. Do not pull your stomach in. Keep your breathing steady and place the tape measure level on your hip bones and at the narrowest part of your waist below your ribs.

Hip: To erect, two legs and close, tape measure around lower abdomen lower margin, in the coxal most prominent place measure a hip circumference

Waist-hip ratio: The ratio of waist circumference to hip circumference

B. Body composition detection

Test instrument: Physical master body composition tester

Body composition test method: same as body weight test method, body fat percentage and lean body weight test results were recorded

C. Quiet heart rate: recorded by morning pulse

D. FMS screening test

Methods: Before the FMS test, the subjects should not do any warm-up activities, and arrange the subjects to perform 7 movements under the guidance of the experimenter. During the test, the participants were given scores based on their performance.

(3) Experimental process

1) Pr-experiment

In order to ensure the safety of the experiment process and effectively reduce the risk of exercise during the experiment, all subjects were checked before the experiment and only those with qualified data could participate in the training, including the test of body shape, body composition, quiet heart rate and FMS and other indicators. Details are as follows:

A. Body shape: body weight, waist circumference, hip circumference, waist-hip ratio, BMI

B. Body composition: body fat percentage, lean body weight and other specific data

C. Quiet heart rate detection: morning pulse test

D. FMS screening: FMS (Functional Movement Screen) is a predictive system that confirms, grades and sorts basic movement patterns, movement limitations and movement asymmetry through a reliable 7-step observation and three exclusion tests

Table 1. Pretest data of FMS indicators of 10 subjects

\begin{tabular}{ccccccccc}
\hline Group & SM & DS & HS & ILL & RS & ASLR & TSPU \\
\hline A & 3 & 2 & 2 & 2 & 2 & $2.2 \pm 0.2$ & $1.4 \pm 0.2$ & $14.4 \pm 0.2$ \\
B & 3 & 2 & $1.8 \pm 0.2$ & 2 & 2 & $2.6 \pm 0.2$ & $1.6 \pm 0.2$ & $14.6 \pm 0.4$ \\
\hline
\end{tabular}

Note: * represents $\mathrm{P}<0.05, * *$ represents $\mathrm{P}<0.01$.SM represents shoulder flexibility; DS stands for squat; HS stands for the hurdle frame step; ILL stands for split back and forth squat; RS stands for rotational stability; ASLR stands for active straight leg knee lift; TSPU stands for trunk stability. 
Table 2. Pretest data of body shape indicators of 10 subjects

\begin{tabular}{ccccccc}
\hline Group & Height & Weight $(\mathrm{kg})$ & BMI $\left(\mathrm{kg} / \mathrm{m}^{2}\right)$ & Waistline $(\mathrm{cm})$ & Hipline $(\mathrm{cm})$ & Waist-hip Ratio, WHR \\
\hline Group A & $159 \pm 2.7$ & $58.58 \pm 3.10$ & $22.92 \pm 0.89$ & $79.32 \pm 1.84$ & $93.50 \pm 1.53$ & $84.40 \pm 1.63$ \\
Group B & $160 \pm 3.1$ & $60.28 \pm 4.48$ & $23.38 \pm 1.15$ & $80.74 \pm 3.05$ & $93.30 \pm 3.49$ & $86.20 \pm 2.69$ \\
\hline
\end{tabular}

Note: * stands for $\mathrm{P}<0.05, * *$ stands for $\mathrm{P}<0.01$.

Table 3. Pretest data for body composition indicators of 10 subjects

\begin{tabular}{cccr}
\hline Groups & & Body Fat Rate $(\%)$ & Lean body mass $(\%)$ \\
\hline Group A & Pr-experiment & $31.96 \pm 1.57$ & $32.12 \pm 0.92$ \\
Group B & Pr-test & $33.72 \pm 1.68$ & $30.42 \pm 0.89$ \\
\hline
\end{tabular}

Note: *represents $\mathrm{P}<0.05, * *$ represents $\mathrm{P}<0.01$.

Table 1, Table 2 and Table 3 showed that there were no significant differences in FMS, body shape, body composition and other related indicators between the two groups (P\&gt; 0.05) and meet the normal distribution, and the physical conditions of the subjects meet the standards of the experiment, the experiment can be carried out.

2) Exercise prescription implementation

A. Time and place

From January to February 2018, it was conducted in "Posture Assessment and Functional Training Studio of Chongqing University" in Siqun Square of Chongqing University. For a total of one month, the training was conducted in the mode of one day rest and one day training every week. Each afternoon training lasted for 90min, including 30min for unified warm-up and relaxation, and 60min for formal training, namely aerobic (40min) and HIIT (20min). Training time: 17:00-18:30 PM.

B. Training content

HIIT: mountain climbing 30s + jumping jacks $40 \mathrm{~s}+$ bear climb 20 meters two back and forth + resistance belt sprint run 30 meters, etc

Intermittent: 60-90 - s

Exercise intensity: target rate $80-90 \%$

Aerobic: continuous jogging in the playground

Exercise intensity: target rate $60 \%-70 \%$

C. Mode of movement

Warm-up: Group A and GROUP B were warmed up by foam axis rolling and dynamic joint pulling

Relaxation: foam axis rolling and static pulling were performed for group A and GROUP B to relax fascia

Training: Group A HIIT+ aerobic group

Schedule: HIIT for 20 minutes followed by cardio for 40 minutes.

Training: Group B aerobic +HIIT group

Schedule: 40 minutes of aerobic training followed by 20 minutes of hilT.

\subsubsection{Mathematical statistics}

Relevant indicators of subjects before and after the experiment were collected, SPSS 22 statistical analysis software was used for statistical analysis, independent sample $\mathrm{T}$ test and paired sample $\mathrm{T}$ test were selected for comparative analysis, and the significance of the differences of indicators between the same group and groups before and after the experiment was determined (significance difference P\&lt; 0.05 and very significant difference. 0.01 ).

\section{Experimental results and analysis}

\subsection{Experimental results and analysis of two training methods on the body shape of obese young women}

Table 4. Data indicators before and after the body shape experiment of young women in group A

\begin{tabular}{cccccccc}
\hline Group & & Height & Weight $(\mathrm{kg})$ & BMI $\left(\mathrm{kg} / \mathrm{m}^{2}\right)$ & Waistline $(\mathrm{cm})$ & Hipline $(\mathrm{cm})$ & Waist Hip rate \\
\hline \multirow{2}{*}{ Group A } & Pr-experiment & $159 \pm 2.7$ & $58.58 \pm 3.10$ & $22.92 \pm 0.89$ & $79.32 \pm 1.84$ & $93.50 \pm 1.53$ & $84.40 \pm 1.63$ \\
& Post-experiment & $159 \pm 2.7$ & $57.56 \pm 3.22 *$ & $22.48 \pm 0.90 *$ & $76.28 \pm 2.75$ & $92.00 \pm 1.77$ & $82.40 \pm 1.86$ \\
\hline
\end{tabular}

Note: *represents $\mathrm{P}<0.05$, **represents $\mathrm{P}<0.01$. 
Table 5. Data indicators before and after the body shape experiment of young women in group B

\begin{tabular}{|c|c|c|c|c|c|c|c|}
\hline & Groups & Height & Weight $(\mathrm{kg})$ & BMI $\left(\mathrm{kg} / \mathrm{m}^{2}\right)$ & Waistline $(\mathrm{cm})$ & Hipline $(\mathrm{cm})$ & Waist hip rate \\
\hline \multirow{2}{*}{ Group B } & Pr-experiment & $160 \pm 3.1$ & $60.28 \pm 4.48$ & $23.38 \pm 1.15$ & $80.74 \pm 3.05$ & $93.30 \pm 3.49$ & $86.20 \pm 2.69$ \\
\hline & Post-experiment & $160 \pm 3.1$ & $59.36 \pm 4.32$ & $21.94 \pm 0.92$ & $78.98 \pm 2.44$ & $92.68 \pm 3.64$ & $85.00 \pm 2.66$ \\
\hline
\end{tabular}

Note: *represents $\mathrm{P}<0.05$, ** represents $\mathrm{P}<0.01$.

Table 6. Data indicators after body shape experiment in young women in groups A and B

\begin{tabular}{|c|c|c|c|c|c|c|c|}
\hline & Group & Height & Weight $(\mathrm{kg})$ & BMI $\left(\mathrm{kg} / \mathrm{m}^{2}\right)$ & Waistline $(\mathrm{cm})$ & Hipline $(\mathrm{cm})$ & WHR \\
\hline Group A & Post-experiment & $159 \pm 2.7$ & $57.56 \pm 3.22$ & $22.48 \pm 0.90$ & $76.28 \pm 2.75$ & $92.00 \pm 1.77$ & $82.40 \pm 1.86$ \\
\hline Grouop B & Post-experiment & $160 \pm 3.1$ & $59.36 \pm 4.32$ & $21.94 \pm 0.92$ & $78.98 \pm 2.44$ & $92.68 \pm 3.64$ & $85.00 \pm 2.66$ \\
\hline
\end{tabular}

Note: *represents $\mathrm{P}<0.05, * *$ represents $\mathrm{P}<0.01$.

\subsubsection{Weight}

(1) Experimental results

The data in Table 4 and 5 show that the weight of all the young women in group A and group B decreased to A certain extent after one-month training. As can be seen from Table 4, the average body weight of subjects in group A decreased from $58.58 \mathrm{~kg}$ to $57.56 \mathrm{~kg}$, A decrease of $1.02 \mathrm{~kg}$, with significant difference $(\mathrm{P}<0.05)$. As shown in Table 5 , the average body weight of group B decreased from $60.28 \mathrm{~kg}$ before the experiment to $59.36 \mathrm{~kg}$, with no significant difference ( $>0.05)$. The comparison of body weight between the two groups after exercise intervention showed that there was no significant difference between "HIIT+ aerobic" and "aerobic +HIIT" under the same conditions $(\mathrm{P}>0.05)$.

(2) Analysis and discussion

Both types of exercise led to weight loss in the young women after a month of trial. The body weight of group A was $58.58 \mathrm{~kg}$ before the experiment and 57.56 after the experiment, and the body weight of group A was $1.02 \mathrm{~kg}$ after the experiment, which was significantly different from that before the experiment $(\mathrm{P}<0.05)$. The body weight of group $\mathrm{B}$ was $60.28 \mathrm{~kg}$ before the experiment and $59.36 \mathrm{~kg}$ after the experiment. The body weight of group B was $0.92 \mathrm{~kg}$ after the experiment. Only from the degree of decline in group A and group B, group A had A significant effect after "HIIT+ aerobic" training. The reasons can be considered from the following aspects. First, from the perspective of energy supply characteristics, the analysis may be that group A "HIIT+ aerobic" directly underwent high-intensity training after dynamic warm-up, mainly atP-CP or glycolysis energy supply system at the beginning of the exercise, consuming A large amount of glycogen substances. When 20 minutes passed, it began to use continuous aerobic exercise for 40 minutes. According to relevant literature, muscle glycogen is mainly consumed in the first 15 minutes of aerobic exercise, and the proportion of fat energy supply starts to increase gradually in the 15-20 minutes after exercise. Does that mean that the following 40 minutes are all fat energy supply, so as to achieve the effect of weight loss? There are also relevant studies proving that aerobic has a good effect on weight loss. Secondly, from the psychological perspective, some relevant literature shows that HIIT can adjust the enthusiasm of training by improving the excitement state. Some relevant articles also point out that long-term aerobic training is monotonous and relatively boring. It is possible that group A "HIIT+ Aerobic" improves the enthusiasm of training after performing HIIT first. For the following aerobic training will be more expected; Third, there was movement experience, when the body composition index integrated with transverse and longitudinal comparison, it was found that the body fat drop in group A was not better than group B, analysis may be done after the HIIT group A because of the increase in positive for aerobic strength set your control more than $60 \%$ to $70 \%$ of the strength of the bull 's-eye rate The training of aerobic endurance did not consume too much fat, but consumed certain glycogen, protein, water, etc., which caused the phenomenon that the weight loss of group A was larger than that of group B, and the fat rate was not as large as that of group B. Although the training methods of the two groups have certain influence on the losing weight, the training method in the experimental group A and group B after no statistical difference, analysis may and short training period, the relationship between the sample size must be rare, but A set of training methods is likely to have movement through the crowd has certain effect on weight loss.

\subsubsection{BMI}

(1) Experimental results

The data in Table 4 and 5 show that after 1 month of exercise intervention, the BMI of all the subjects in group A and group B decreased to A certain extent. As can be seen from Table 4, the average BMI of the subjects in group A decreased 
from 22.92 to 22.48, with A significant difference $(\mathrm{P}<0.05)$. As can be seen from Table 5, the average BMI of subjects in group B decreased from 23.38 to 21.94, with no significant difference ( $\mathrm{P}>0.05)$. BMI comparison between the two groups after exercise intervention showed that there was no significant difference between the two exercise modes under the same conditions $(\mathrm{P}>0.05)$.

(2) Analysis and discussion

Both types of exercise reduced the BMI of the young women after a month of study. Compared with the same group, THE BMI of group A decreased from 22.92 before the experiment to 22.48 after the experiment, showing significant difference $(\mathrm{P}<0.05)$. Group B decreased from 23.38 before the experiment to 21.94 after the experiment, showing no significant difference $(\mathrm{P}>0.05)$. It indicated that the comparison of "HIIT+ aerobic" in BMI in group A before and after training was more significant $(\mathrm{P}<0.05)$, while there was no significant difference between the two groups after the experiment $(\mathrm{P}>0.05)$, which may be due to the short exercise cycle and small sample size.

\subsubsection{Waistline, hip and waist-to-hip ratio}

(1) Experimental results

The data in Table 4 and 5 showed that waist circumference, hip circumference and waist-hip ratio of subjects in group A and group B all showed A decreasing trend after 1 month of exercise intervention. Table 4 and Table 5 showed that although waist circumference, hip circumference and waist-hip ratio of group A decreased to A certain extent, there were no significant differences compared with those after the experiment $(\mathrm{P}>0.05)$. In group $\mathrm{B}$, there was a decrease after exercise intervention compared with before intervention, but there was no significant difference $(\mathrm{P}>0.05)$. There was no significant difference in waist circumference, hip circumference and waist-hip ratio between the two groups, which was comparable between the two groups. After intervention, there was no significant difference in body shape between the two groups ( $\mathrm{P}>0.05)$. The body weight and BMI of HIIT+ aerobics in group A were significant $(\mathrm{P}<0.05)$. There was no significant difference in body shape index of "aerobic + HIIT" in group B $(\mathrm{P}>0.05)$.

(2) Analysis and discussion

After passing 1 month experiment, two kinds of motion means have certain drop range to waist circumference, buttock circumference and waist buttock ratio. Waist circumference of group A was $79.32 \mathrm{~cm}$ before the experiment, and decreased by $3.4 \mathrm{~cm}$ after the experiment; waist circumference of group B was $80.74 \mathrm{~cm}$ before the experiment, and decreased by 1.76 after the experiment; Studies of HIIT on abdominal fat showed that high-intensity interval exercise significantly reduced the total fat and abdominal fat of subjects; It is also possible that core exercises such as planking, crawling and climbing can be used in the design of exercise programs to strengthen the core muscles, tighten the slack abdominal muscles and reduce the dimension of the waist. The hip circumference of group A was $93.5 \mathrm{~cm}$ before the experiment and decreased by $1.5 \mathrm{~cm}$ after the experiment, and that of group B was 93.3 before the experiment and decreased by 0.62 after the experiment. The waistto-hip ratio of group A was 84.4, which decreased by 2 after the experiment, and that of group B was 86.2 before and 1.2 after the experiment. From the above overall numerical comprehensive comparison of the experimental group A than in group B after decline after the experimental effect is good, shows that the training method of group A than group B training methods in waist circumference, hip circumference, waist-to-hip ratio may have certain effect, but from A statistical perspective, compared the effect of the two methods after training, no significant difference $(\mathrm{P}>0.05)$, The analysis may be influenced by short training period and small sample size.

\subsection{Experimental results and analysis of body composition of obese young women by two training methods}

Table 7. Data indicators before and after the body composition of young women in group A

\begin{tabular}{|c|c|c|c|}
\hline \multicolumn{2}{|c|}{ Group } & \multirow{2}{*}{$\begin{array}{c}\text { Body Fat Rate }(\%) \\
31.96 \pm 1.57\end{array}$} & \multirow{2}{*}{$\begin{array}{c}\text { Lean body mass }(\%) \\
32.12 \pm 0.92\end{array}$} \\
\hline Groun 4 & Pr-experiment & & \\
\hline Uivoup & Post-experiment & $30.70 \pm 1.08$ & $32.68 \pm 0.65$ \\
\hline
\end{tabular}

Note: *represents $\mathrm{P}<0.05, * *$ represents $\mathrm{P}<0.01$.

Table 8. Data indicators before and after the body composition of young women in group B

\begin{tabular}{|c|c|c|c|}
\hline \multicolumn{2}{|c|}{ Group } & Body Fat Rate (\%) & Lean Body Mass $(\%)$ \\
\hline \multirow{2}{*}{ Group B } & Pr-experiment & $33.72 \pm 1.68$ & $30.42 \pm 0.89$ \\
\hline & Post-experiment & $30.84 \pm 1.12$ & $31.66 \pm 0.92$ \\
\hline
\end{tabular}

Note: *represents $\mathrm{P}<0.05$, **represents $\mathrm{P}<0.01$. 
Table 9. Data indicators after the body composition of young women in groups A and B

\begin{tabular}{|c|c|c|c|}
\hline \multicolumn{2}{|c|}{ Group } & Body Fat Rate (\%) & Lean Body Mass (\%) \\
\hline Group A & Post-experiment & $30.70 \pm 1.08$ & $32.68 \pm 0.65$ \\
\hline Group B & Post-experiment & $30.84 \pm 1.12$ & $31.66 \pm 0.92$ \\
\hline
\end{tabular}

Note: *represents $\mathrm{P}<0.05$, **represents $\mathrm{P}<0.01$.

\subsubsection{Body fat rate}

The data in Table 7 and 8 showed that after one-month exercise intervention, the body fat percentage of all the subjects in Group A and Group B decreased to A certain extent. As can be seen from Table 7, the average body fat percentage of subjects in group A decreased from $31.96 \%$ to $30.70 \%$, with no significant difference $(\mathrm{P}>0.05)$. As can be seen from Table 8 , the average body fat percentage of all subjects in group B decreased, but there was no significant difference $(\mathrm{P}>0.05)$. As shown in Table 9, there was no significant difference between group A and group B after the experiment $(\mathrm{P}>0.05)$.

\subsubsection{Lean body mass}

The measured muscle content of young women in group A and group B before and after the experiment are shown in Table 7 and Table 8. Before experimental intervention, there was no significant difference in lean body weight between the two groups, which was comparable between groups. After exercise intervention, the average lean body weight of group A was increased, but there was no significant difference $(\mathrm{P}>0.05)$. The mean lean body weight of group $\mathrm{B}$ was increased without significant difference $(\mathrm{P}>0.05)$. As shown in Table 9, there was no significant difference between group A and group $\mathrm{B}$ after the experiment $(\mathrm{P}>0.05)$.

Body fat percentage is the percentage of body fat as a percentage of body weight. It is a measure and assessment of the body's degree of obesity and health risks. The higher the body fat content, the higher the body fat percentage. Too much body fat can lead to obesity, and conversely, too little body fat can also affect people's health. From a health point of view, adult male fat percentage is usually between $10 \%$ and $25 \%$ and female fat percentage is between $18 \%$ and $30 \%$. Adult male fat percentage $>25 \%$ or female fat percentage $>30 \%$ is usually defined as obese as shown in Table 10 .

Table 10. Body fat percentage evaluation criteria

\begin{tabular}{|c|c|c|c|c|c|c|}
\hline Gender & Age Bracket & Much less & Better & Normal & More & Redundancy \\
\hline \multirow{4}{*}{ Male } & $20-29$ & $<3$ & $13-18$ & $18.1-23$ & $23.1-28$ & $\geq 28.1$ \\
\hline & $30-39$ & $<3$ & $14-19$ & $19.1-24$ & $24.1-29$ & $\geq 29.1$ \\
\hline & $40-49$ & $<3$ & $15-20$ & $20.1-25$ & $25.1-30$ & $\geq 30.1$ \\
\hline & $\geq 50$ & $<3$ & $16-21$ & $21.2-26$ & $26.1-31$ & $\geq 31.1$ \\
\hline \multirow{2}{*}{ Female } & $40-49$ & $<12$ & $20-25$ & $25.1-30$ & $30.1-35$ & $\geq 35.1$ \\
\hline & $\geq 50$ & $<12$ & $21-26$ & $26.1-31$ & $31.3-36$ & $\geq 36.1$ \\
\hline
\end{tabular}

According to the one-month experiment, the body fat percentage of group A was $31.96 \%$ before the experiment, but decreased to 30.70 after exercise intervention. Group B of body fat rate has dropped from 33.72 to 30.84 , the single from the point of fall, the effect of group B than in group A is better, the body fat rate and lean body mass index, lean body mass in group A than group B growth more, less body fat analysis of group B and group B thin weight gain, because the increase in lean body mass can effectively improve human basal metabolism, and thus effectively burning fat. Although the body fat percentage of both groups decreased, there was no significant difference between the two groups from a statistical point of view $(\mathrm{P}>0.05)$. It was possible that the subjects had sports experience, and the training cycle was small and the sample size was small, so there was no statistically significant difference. Therefore, "HIIT+ aerobic" in group A and "aerobic +HIIT" in group B had no significant effect on the decline of body fat percentage, but "aerobic +HIIT" in group B may have some influence on the decline of body fat percentage in young women with exercise experience.

Lean body mass, also known as "fat loss weight," is commonly used to assess muscle mass in situations where precise measurements of muscle mass are not possible. After A month of experiment, it can be found that the average lean body weight of group A and group B has A small increase. Some articles show that: High-intensity exercise leads to the increase of body lean body weight. From the increase range, it was found that the increase of average lean body weight in group B was better than that in group A, and it was also found that the decrease of average body fat in group B was more than that in group A, because the increase of average lean body weight in group B affected the decrease of average body fat in 
group B. However, there was no significant difference in average lean body weight between group A and group B ( $\mathrm{P}>0.05)$. The subjects may be people who have sports experience. Due to the small training period and small sample size, there is no statistically significant difference. Therefore, "HIIT+ Aerobic" in group A and "aerobic +HIIT" in group B have no significant effect on the increase of lean body weight, but "aerobic +HIIT" in group B may have some influence on the increase of lean body weight in young women with exercise experience.

\subsection{Experimental results and analysis of FMS in obese young women by two training methods}

Table 11. Comparison of FMS indicators between subjects in group A and group B before and after experiment

\begin{tabular}{ccccccccccc}
\hline & Group & SM & DS & HS & ILL & RS & ASLR & TSPU & Total \\
\hline \multirow{2}{*}{ Group A } & Pr-experiment & 3 & 2 & 2 & 2 & 2 & $2.2 \pm 0.2$ & $1.4 \pm 0.24$ & $14.4 \pm 0.2$ \\
& Post-experiment & 3 & 2 & 2 & 2 & 2 & $2.2 \pm 0.2$ & 2 & $15.2 \pm 0.2$ \\
\multirow{2}{*}{ Group B } & Pr-experiment & 3 & 3 & $1.8 \pm 0.2$ & 2 & 2 & $2.6 \pm 0.2$ & $1.6 \pm 0.2$ & $14.6 \pm 0.4$ \\
& Post-experiment & 3 & 3 & 2 & 2 & 2 & $2.6 \pm 0.2$ & 2 \\
\hline
\end{tabular}

Note: * represents $\mathrm{P}<0.05$, ** represents $\mathrm{P}<0.01$.SM represents shoulder flexibility; DS stands for squat; HS stands for the hurdle frame step; ILL stands for split back and forth squat; RS stands for rotational stability; ASLR stands for active straight leg knee lift; TSPU stands for stable torso push-up.

(1) Experimental results

The indicators of FMS before and after the experiment are shown in Table 11. Before intervention, there was no significant difference in FMS between group A and group $\mathrm{B}(\mathrm{P}>0.05)$. After experimental intervention, there was no significant difference in SM, DS, HS, ILL, RS and ASLR in group A ( $>>0.05)$. Although TSPU (push up) was significantly improved to 2 points, there was no statistically significant difference $(\mathrm{P}>0.05)$. The same was true for group $\mathrm{B}$; There was no significant difference in TSPU between the two groups $(\mathrm{P}>0.05)$, but TSPU of the two groups improved after training.

(2) Analysis and discussion

Functional Movement Screen (FMS) is a screening assessment tool that focuses on assessing whether the flexibility and stability of shoulder, spine, core, hip, knee and ankle joints meet the needs of complex movements. The total FMS score was 21. Overall scores are commonly used to determine injury risk, and studies have shown that a score of less than 14 significantly increases an athlete's risk of serious injury.

As can be seen from Table 11, there was no significant difference between the two groups before training $(\mathrm{P}>0.05)$. After training, there was no significant difference between group A and group B ( $>0.05)$, no matter within the same group or between groups. However, it was found that the score of TSPU (trunk stable push-ups) in group A and GROUP B increased from 1 point to 2 points. The reasons for the poor performance of TSPU in the test are poor reflective core stability, and the two training methods are both improved to 2 points, indicating that the core stability has been improved to some extent. The analysis may be related to the analysis of the results of FMS screening when designing HIIT training movements, and some core functional movements such as: Crawling, plank, mountain running and other high-impact movements were intermixed, and combined training was conducted in accordance with the principle of HIIT high-intensity intervals. Therefore, TSPU (stable trunk push up) of some subjects improved after the experiment.

\subsection{Experimental results and analysis of two training methods on quiet heart rate of obese young women}

Table 12. Test data of patients before and after the test of resting heart rate in group $A$ and group $B$

\begin{tabular}{ccc}
\hline Heart rate of rest & Group A & Group B \\
\hline Pr-experiment & $61.60 \pm 0.92$ & $62.00 \pm 1.26$ \\
Post-experiment & $58.80 \pm 1.42^{*}$ & $61.00 \pm 1.095$ \\
\hline
\end{tabular}

Note: *represents $\mathrm{P}<0.05$, **represents $\mathrm{P}<0.01$.

(1) Experimental results

Indicators of resting heart rate before and after the experiment are shown in Table 12. Before intervention, there was no significant difference in resting heart rate between the two groups $(\mathrm{P}>0.05)$. After experimental intervention, group $\mathrm{A}$ 
showed significant difference in quiet heart rate after training compared with before training $(\mathrm{P}<0.05)$, while group $\mathrm{B}$ showed no significant difference in quiet heart rate after training $(\mathrm{P}>0.05)$. Compared with group $\mathrm{B}$, group A had more significant difference in resting heart rate after training $(\mathrm{P}<0.05)$.

(2) Analysis and discussion

After 4 weeks of exercise intervention, group A and group B were declined, this may and this month regular insist on high intensity exercise is associated with the exercise of sustainability, on the one hand, games affect control weakened heart activity of sympathetic nerve, the increased vagus nerve, resulting in A loss of resting heart rate and makes the heart rate increases in reserve, The pumping capacity of the heart improved; On the other hand, exercise can enhance the ventricular systolic force of the heart, so that the ventricular cavity becomes larger, the heart will show an increase in output per wave, and the heart rate slows down. Group A showed significant difference in resting heart rate after training $(\mathrm{P}<0.05)$. Compared with group $\mathrm{B}$, group A had more significant difference $(\mathrm{P}<0.05)$. It indicates that group A and group B both have A certain effect on the quiet heart rate, and group A has A more significant effect on the quiet heart rate.

\section{Conclusions and recommendations}

\subsection{Conclusions}

After 1 month of exercise intervention:

(1) Two different sequential combination training methods had certain effect on fat reduction in obese female youth who had exercise experience, but there was no significant difference between the two methods in fat reduction effect $(\mathrm{P}>0.05)$.

(2) There was no significant difference in the improvement of joint function in obese young women with exercise experience $(\mathrm{P}>0.05)$, but there was a certain effect on the improvement of core function.

(3) The two different sequential combination training methods had A certain effect on the reduction of resting heart rate in obese women who had exercise experience, group A was better than group B, and there was A significant difference between the two groups $(\mathrm{P}<0.05)$.

\subsection{Recommendations}

From the training effect, it was preliminarily confirmed that the two training methods had A certain effect on overweight or obese young women with exercise experience. "HIIT+ aerobic" in group A was better than "aerobic +HIIT" in group B, and some indexes showed significant differences. However, the training effect of the two methods on women with exercise experience and mild obesity degree, as well as other women of different ages and men with different age and obesity degree, is still worth further study.

In addition, the sample size of this study is too small and the training period is too short. It is suggested to increase the sample size and training period when conditions are available, which may reflect the experimental effect more clearly and provide more meaningful reference materials for relevant studies.

\section{References}

[1] Ogden C L, Carroll M D, Fryar C D, et al. Prevalence of Obesity Among Adults and Youth: United States, 20112014[J]. NCHS DATA Brief, 2015, (219): 1-8.

[2] Li J L. Experimental study on the effect of "aerobic + anaerobic exercise" on weight loss of obese Female college students[D]. Jilin: Jilin University, 2018.

[3] Wang Juan, Wang Zhengzhen, Luo Xijuan, et al. Effect of high-intensity interval training on the risk of cardiovascular disease in type 2 diabetes mellitus[J]. Chinese journal of sports medicine, 2016, 35(6): 561-56.

[4] Notta VF, Aguila MB, Mandarim-DE-Lacerda CA. High-in-tensity interval training (swimming) significantly improves the adverse metabolism and comorbidities in diet-in-duced obese mice[J]. Sports Med Phys Fitness, 2016, 56(5): 655-663.

[5] Mackay J, Greenlund K, Mendis S, et al. The Atlas of Heart Disease and Stroke. World Health Organization, Geneva, Switzerland, 2004.

[6] NG M, FLEMING T, ROBINSON M, et al. Global, regional, and National prevalence of overweight and obesity in children and adults during 1980-2013: a systematic analysis for the Global Burden of Disease Study 2013[J]. Lancet, 2014, 384(9945): 766-781.

[7] Jiang Yong. Study on the prevalence of overweight and obesity in Adults in China[D]. Beijing: Chinese Center for Disease Control and Prevention, 2013.

[8] Chen Yishan, Zhou Yue, Yu Liang, et al. The influence of overweight and obesity on cardiac function in children aged 
8 9 years[J]. Chinese School Health, 2017, (09): 1291-1295.

[9] Hales C. M., Carroll M. D., Fryar C. D., et al.Prevalence of obesity among adults and youth[M]. United States, NCHS Data Brief, 2017, 288: 1-8.

[10] Healy M. Americans keep piling on the pounds[J]. The Dallas Morning News, 2017, 14(10): 7A.

[11] Ward Z. Simulation of growth trajectories of child-hood obesity into adulthood[J]. New England Journal of Medicine, 2017, 377: 2145-2153.

[12] Sogou Encyclopedia. Eat medicine [EB/OL]. http://baike.sogou.com/v7983793.

[13] Zhang Yan, Zhang Xiansong. Female body shaping strategy[M]. Wuhan: China University of Geosciences Press, 2015:190.

[14] BILLATL V. Interval Training for Performance: A scientific and Empirical Practice special recommendations for middle- and long- distance running. Part I: Training of Aerbic interval training[J]. Sports Med, 2001, 31(1): 13-31.

[15] Li Shuguang. Nutrition and Food Hygiene[M]. People's Medical Publishing House, 2012.

[16] Billat L V. Interval training for performance: A scientific and empirical practice[J]. Sports Medicine, 2001, 31(1): 13-31.

[17] Dong Lei. Effects of high-intensity interval training on myocardial oxygen consumption during exercise in young men[D]. Changsha: Xiangya Hospital, 2012.

[18] Ingul C B, Tjonna A E, Stolen T O, et al. Impaired cardiac function among obese adolescents; effect of aerobic intercal training[J]. Arch Pediatr Adolesc Med, 2010, 164(9): 852-859.

[19] Zhang Hongying. Treatment of 46 cases of simple obesity with acupuncture[R]. Jilin Journal of Traditional Chinese Medicine, 2004:34.

[20] Kenneth H.Cooper (USA), Zhu Weimo (translated). The history of aerobic exercise -- 50 years and still in progress[J]. Sports research, 2018, 39 (6).

[21] Tremblay A, Despres, J P, Leblanc C, et al. Effect of intensity of physical activity on body fatness and fat distribution[J]. The American Journal of Clinical Nutrition, 1990, 51(2):153.

[22] Xie Chao, Jin Chengji, Zhang Jun. Effect of aerobic exercise on obesity in Chinese children: A meta-analysis[J]. Journal of Nanjing University of Physical Education (Social Science Edition), 2016, (02): 84-90.

[23] Yin Haojin. Effects of water jogging on body composition, lipid metabolism and regulatory hormones in obese people[D]. Beijing: Beijing Sport University, 2007.

[24] Qi Yugang, Huang Jinhong, TAN Sijie. Effects of HIIT and Continuous aerobic exercise on weight loss in Obese Female College students[J]. China sports science and technology, 2013, 49(1): 30-33.

[25] Chen Zhuo. The effect of square dancing on the fitness of overweight and obese women aged 55-60 years in Beilin District, Xi 'an City[D]. Xi 'an: Xi 'an Institute of Physical Education, 2016.

[26] Zhang Lian-qing. Study on the influence of different exercise patterns on obesity in young men[D]. Changchun: Northeast Normal University, 2011.

[27] Reindell H, Roskamm H. Ein Beitrag zu den physiologischen Grundlagen des Intervall training unter besonderer Berucksichtigung des Kreilaufes[J]. Scheweiz Z Sportmed, 1959, 7: 1-8.

[28] THOMPSON P. Break through the speed barrier with the "new interval training”[J]. Athl Wkly, 2005, 59: 62-63.

[29] Ingul C B,Tjonna A E,Stolen T O,et al. Impaired cardiac function among obese adolescents; effect of aerobic intercal training[J]. Arch Pediatr Adolesc Med, 2010, 164(9): 852-859.

[30] Schjerve I, Tyldum G, Tjønna A, et al. Both aerobic endurance and strength training programmes improve cardiovascular health in obese adults[J]. Clinical Science, 2008, 115(9): 283-293.

[31] Warburton D E, McKenzie D C, et al. Effectiveness of high-intensity interval training for the rehabilitation of patients with coronary artery disease[J]. Am J Cardiol, 2005, 95(9): 1080-1084.

[32] Bartlett J D, Close G L, Maclaren D P M, et al. High-intensity interval running is perceived to be more enjoyable than moderate-intensity continuous exercise: implications for exercise adherence[J]. Journal of sports sciences, 2011, 29(6): 547-553.

[33] Ciolac E G, Bocchi E A, Bortolotto L A, et al. Effects of high-intensity aerobic interval training vs. moderate exercise on hemodynamic, metabolic and neuro-humoral abnormalities of young normotensive women at high familial risk for hypertension[J]. Hypertension Research, 2010, 33(8): 836-843.

[34] Nybo L, Sundstrup E, Jakobsen M D, et al. High-Intensity Training versus Traditional Exercise Interventions for Promoting Health[J]. Medicine \& Science in Sports \& Exercise, 2010, 42(10): 1951-1958.

[35] Hood M S, Little J P, Tarnopolsky M A, et al. Low-Volume Interval Training Improves Muscle Oxidative Capacity in Sedentary Adults[J]. Medicine \& Science in Sports \& Exercise, 2011, 43(10): 1849-1856.

[36] Tsekouras, Magkos Y E, Kellas F, et al. High-intensity interval aerobic training reduces hepatic very low-density lipoprotein-triglyceride secretion rate in men[J]. Am J Physiol Endocrinol Metab, 2008, 295(4): E851.

[37] BalaM J, Little J P, Macdonald M J, et al. Physiological adaptations to low-volume,high-intensity interval training in health and disease[J]. Journal of Physiology, 2012, 59(5).

[38] Ibala M J,Megee S L. Metabolic adaptations to short-term high-intensity interval trainning:a little pain for a lot of 
gain?[J]. Exercise \& Sport Sciences Revives, 2008, 36(2).

[39] Dong Lei. Effects of high-intensity interval training on myocardial oxygen consumption during exercise in young men[D]. Changsha: Xiangya Hospital, 2012.

[40] Sijie T, Hainai Y, Fengying Y, et al. High intenval exercise training in overweight young women[J]. Sports Med Phys Fitness, 2012, 52(3): 255-262.

[41] Saris W H M, Schrauwen P. Substrate oxidation differences between high- and low-intensity exercise are compensated over 24 hours in obese men[J]. International Journal of Obesity, 2004, 28(6): 759-765.

[42] Major GC, Piche ME, Bergeron J, et al. Energy expenditure from physical activity and the metabolic risk profile at menopause[J]. Med Sci Sports Exerc, 2005, 37(2): 204-212.

[43] Wang Xinbo. Comparative study on fat Reduction Effect of High-Intensity Intermittent Exercise and Aerobic Exercise[D]. Jilin University of Physical Education, 2014.

[44] Xi Lu, Zhang Xiang. Review on the Effect of Intermittent High-Intensity Training on Weight Loss[J]. Sports Science and Technology, 2016, (06): 33-34+38.

[45] Smith-Ryan A E, Trexler E T, Wingfield H L, et al. Effects of high-intensity interva training on cardiometabolic risk factors in overweight/obese women[J]. Journal of Sports Sciences, 2016, 34(21): 2038-2046.

[46] Paoli A, Pacelli Q F, Moro T, et al. Effects of high-intensity circuit training, low-intensity circuit training and endurance training on blood pressure and lipoproteins in middle-aged overweight men[J]. Lipids in Health \& Disease, 2013, 12(1): 131.

[47] Tjonna A E, Stolen T O, Bye A, et al. Aerobic interval training reduces cardiovascular risk factors more than a multitreatment approach in overweight adolescents[J]. Clinical Science, 2009, 116(4): 317-326.

[48] Sawyer B J, Tucker W J, Bhammar D M, et al. Effects of high-intensity interval training and moderate-intensity continuous training on endothelial function and cardiometabolic risk markers in obese adults[J]. Journal of Applied Physiology, 2016, 121(1):279

[49] Wang Mingxian, Li Yuzhou. Effects of Aerobic Exercise and Strength Training on Physical Fitness of Overweight College Students[J]. Journal of Inner Mongolia Normal University, 2014, 43(2): 259-260.

[50] Ren Q. A prospective study on the appropriate cut point value of obesity evaluation index for predicting hypertension in Chinese adults[D]. Chinese Center for Disease Control and Prevention, 2016.

[51] Chen Tongqiang. Discussion on the new viewpoint of weight loss in HIIT[J]. Contemporary Sports Science and Technology, 2014(13): 11-12.

[52] Wang Jun, Wang Ruiyuan, Tian Jiming. Study on the effect of intermittent exercise on weight loss[J]. Journal of Guangzhou Sport University, 2007, 27(1): 100-101.

[53] Bryner R W, Toffle R C, Ullrich I H, et al. The effects of exercise intensity on body composition, weight loss, and dietary composition in women[J]. Journal of the American College of Nutrition, 1997, 16(1): 68-73.

[54] Major GC, Piche ME, Bergeron J, et al. Energy expenditure from physical activity and the metabolic risk Profile at menopause[J]. Med Sci Sports Exer, 2005, 37(2): 12-204.

[55] Zhang Lianqing. Study on the influence of different exercise patterns on obesity in young men [D]. Changchun: Northeast Normal University, 2011.

[56] Cui Can. Study on the influence of aerobic and core strength training on the exercise prescription of middle-aged women with central obesity[D]. Tianjin: Tianjin Institute of Physical Education, 2015.

[57] Chen Qiong, Cao Jie, Zhao Lijun et al. Effects of different exercise patterns on body composition, inflammatory factors and exercise ability of obese adolescents[J]. Journal of Jilin University (Medical Science Edition), 2015(05): 10701075.

[58] Li J L. Study on the effect of aerobic and anaerobic exercise on weight loss of obese female college students[D]. Jilin: Jilin University, 2018.

[59] Zhang D. An empirical study on the effect of resistance training combined with aerobic exercise on obesity and weight loss in young women[D]. Jiangxi: Jiangxi Normal University, 2014.

[60] Fan Baohui. Effect of intermittent high-intensity exercise on weight loss in obese young men[D]. Shanghai: Shanghai Normal University, 2018.

[61] Ren Qian. A prospective study on the appropriate pointcut value of obesity evaluation index for predicting hypertension in Chinese adults[D]. Chinese Center for Disease Control and Prevention, 2016.

[62] Qi Yugang, Huang Jinhong, Tan Sijie. Effects of HIIT and Continuous aerobic exercise on weight loss in Obese Female College students[J]. China sports science and technology, 2013, 49(1): 30-33.

[63] Wang Jun, Wang Ruiyuan, Tian Jiming. Study on the effect of intermittent exercise on weight loss[J]. Journal of Guangzhou Institute of Physical Education, 2007, (01): 99-101.

[64] Wang Jingjing, et al. The effect of 12-week high-intensity interval training on abdominal fat in obese young women[D]. Hebei: Hebei Normal University, 2013. 